\section{NEXT GENERATION RECALLS AND REMINDERS}

iSmile have developed not only an automated recall and reminder feature, but a system which goes beyond simple automation. Clever three-step automation now allows you to carry on with your day-to-day jobs while iSmile quietly targets patients in your database that you may not have seen in your practice for some time. Admin users can set parameters for every stage of the automated recall process and also have the ability to check how the automation is performing in real terms.
iSmile's three step automation can be used for contacting patients that haven't been into your practice for some time using a combination of letters, emails and SMS messages. The extensive reporting features allow you to keep a handle on budgets for these processes too.

Speak to one of iSmile's advisors on 08454681287 to see how clever automation could bring you more patient bookings while keeping costs to a minimum.

Visit www.ismiledental.co.uk.

\title{
ACHIEVE UNSURPASSED ORAL HYGIENE
}

To achieve an unsurpassed level of oral hygiene leading to excellent gum health, the challenge is to eliminate odour causing volatile sulphur compounds (VSCs) and bacteria associated with the build-up of plaque, tooth decay and gum problems. Working closely with the natural oral $\mathrm{pH}$, the UltraDEX Performance Oral Care range could be of value to a wide spectrum of oral hygiene concerns when used as part of an oral hygiene regime. UltraDEX bridges the gap between professional and home care, increases patient compliance and helps to enhance treatment outcomes. Backed by scientific research, the clinically proven technology within UltraDEX protects against plaque, restores natural whiteness and eliminates bad breath instantly for at least 12 hours. Specialised UltraDEX Oral Healthcare Kits are available, which can be personalised with practice logo and details. The kits offer outstanding value for money and are a convenient way to increase practice profile, patient compliance and revenue. Dental professionals often incorporate UltraDEX into their treatment plan. For further information visit www.periproducts.co.uk or email dental@periproducts.co.uk.

\section{BLACK TOOTHPASTE FOR WHITER TEETH}

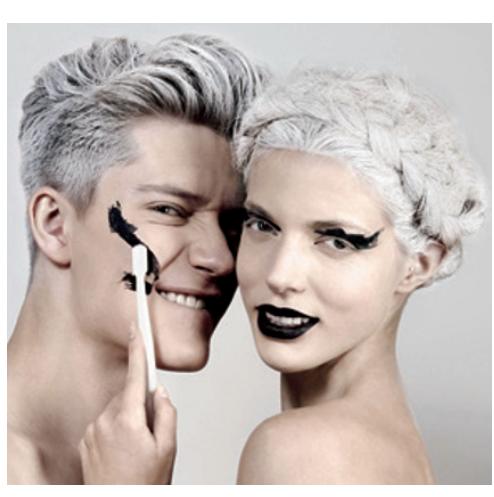

As an oral healthcare specialist, Curaprox understands just how important it is to provide safe and effective solutions to those that need it the most.

With DIY dentistry methods used by many as an alternative - albeit dangerous and unproven - the Black Is White toothpaste from Curaprox is a safer, effective and more cost effective solution to tooth whitening. Using a 15,000 ppm hydroxyapatite layer to protect the enamel and a physical blue filter to make the teeth appear optically whiter, your patients can now experience both a brighter smile and better oral health.

Essentially, by using the key ingredient, activated carbon, and its natural qualities, this cutting-edge toothpaste can remove discolouration without abrading or bleaching.

To find out how you can offer this wonderfully black toothpaste to give your patients the white smile they deserve, contact Curaprox today.

For more information call 01480 862084, emailinfo@curaprox.co.uk or visit www.curaprox.co.uk.

\section{ONE-DAY INTRO TO DENTAL SLEEP MEDICINE}

The British Society of Dental Sleep Medicine (BSDSM) is again holding its popular 'Introductory Course in Dental Sleep Medicine' event for dentists and dental technicians on Saturday 18 June at Leicester Royal Infirmary.

This course enables you to integrate dental sleep The BSDSM President, Dr medicine (DSM) in your Aditi Desai, who will be practice, treat those of your leading the course patients who snore, and market the treatment as a service to potential new patients. To assess the likely demand for DSM, simply add this question to your routine medical health questionnaire: Do you or your partner snore?

The course is from 9.00 am to $5.15 \mathrm{pm}$ and qualifies for six hours of verifiable CPD. The cost of £298 for BSDSM members and $€ 350$ for non-members includes lunch and refreshments. Book early as these courses get over-subscribed.

For more information and online booking go to: http://dentalsleepmed.org.uk/shop/.

\section{MORE POWERFUL THAN CONVENTIONAL FLUORIDE}

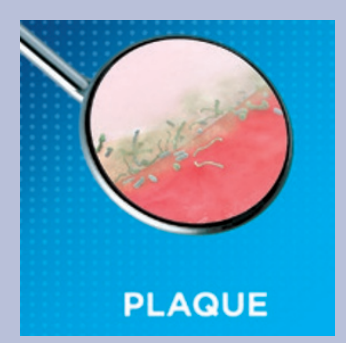

The positive effects of fluoride-containing toothpastes as a defence against dental caries are well documented. Stannous fluoride was the first scientifically recognised fluoride and in recent years has been stabilised and com-

bined with sodium hexametaphosphate, to provide additional protection.

Stabilised stannous fluoride protects teeth against caries in two ways. Firstly, it strengthens enamel and dentine to inhibit demineralisation and promote remineralisation. Secondly, it has the ability to reduce the incidence of Streptococcus mutans. Thus the anti-caries effect of such formulations includes a combination of physical chemical effects and antimicrobial actions.

Oral-B Pro-Expert toothpaste uses stabilised stannous fluoride, which is more powerful than conventional fluoride. ${ }^{1}$ In laboratory studies, when compared with conventional fluoride toothpaste, subjects showed significantly fewer caries increments. For more information or samples contact your Oral-B representative.

1. Stookey G K, Mau M S, Isaacs R L, Gonzalez-Gierbolini C, Bartizek $R D$, Biesbrock $A R$. The relative anticaries effectiveness of three fluoride-containing dentifrices in Puerto Rico. Caries Res 2004; 38: 542-550. 